\title{
Gestão da cadeia de suprimentos de empresas metalmecânicas do estado de Minas Gerais
}

A gestão da cadeia de suprimentos (GCS) é uma vertente da administração muito complexa de se controlar, pois, em geral, deve-se gerir pessoas, produtos, serviços, informações, instalações, produção, centros de distribuição, relação com fornecedores e relação com clientes. Além disso, a cadeia também está inserida em vários outros ambientes sistêmicos. Desta forma, o objetivo deste estudo é analisar o impacto das práticas de GCS sobre o diferencial competitivo e o desempenho organizacional. Mediante a capacidade das empresas integrarem à suas redes de relacionamentos comerciais levanta-se o seguinte problema: Qual o impacto das práticas de GCS sobre o diferencial competitivo e o desempenho organizacional? Para responder à pergunta, foi estudado o contexto das empresas metalmecânicas do estado de Minas Gerais. A pesquisa é de natureza aplicada, com abordagem quantitativa, utilizando a Modelagem de Equações Estruturais como procedimento técnico de análise através de coletas de dados tipo survey. Os dados do estudo foram colhidos a partir de 269 empresas para testar três hipóteses. Os resultados de correlação indicam que os níveis mais altos de práticas de GCS podem levar ao diferencial competitivo melhor, mas estas mesmas práticas não impactam diretamente sobre o desempenho organizacional. Além disso, as correlações indicam que o diferencial competitivo tem um impacto direto e positivo sobre o desempenho organizacional.

Palavras-chave: Gestão da Cadeia de Suprimento; Diferencial Competitivo; Desempenho organizacional.

\section{Supply chain management of mechanical metal companies of the Minas Gerais state}

\begin{abstract}
Supply chain management (GCS) is a very complex branch of management to control because, in general, people, products, services, informations, facilities, production, distribution centers, supplier relationships, and customer relationships should be managed. Furthermore, the supply chain is also included in several other systemic environments. In this way, this study aims to analyze the impact of the SCM practices on competitive differential and organizational performance. Through the capacity of companies, integrate to their commercial relationships network raises the following problem: What is the impact of the SCM practices on the competitive differential and organizational performance? To answer this question, the context of mechanical metal companies of the Minas Gerais state was studied. The research is of an applied nature, with a quantitative approach, using a Structural Equation Modeling as a technical procedure for analysis through surveys. Data from the study were collected from 269 companies to test three hypotheses. Correlation results indicate that higher levels of SCM practices may lead to a better competitive differential, but these same practices do not directly impact organizational performance. Also, competitiveness can have a direct and positive impact on organizational performance.
\end{abstract}

Keywords: Supply Chain Management; Competitive Advantage; Organizational Performance.

Topic: Sistemas Logísticos

Reviewed anonymously in the process of blind peer.
Received: 07/01/2021

Approved: 23/03/2021
Eliane Santos Vargas (D)

Universidade Federal do Rio de Janeiro, Brasil

http://lattes.cnpq.br/2055578741412368

http://orcid.org/0000-0002-4639-0951

elianevargas84@gmail.com

\section{Elisangela Martins de Sá (iD}

Centro Federal de Educação Tecnológica de Minas Gerais, Brasil

http://lattes.cnpq.br/4686246805500174

http://orcid.org/0000-0002-7801-0087

elisangela.martinss@gmail.com
Referencing this:

VARGAS, E. S.; SÁ, E. M.. Gestão da cadeia de suprimentos de empresas metalmecânicas do estado de Minas Gerais. Revista Brasileira de Administração Científica, v.12, n.1, p.277-290, 2021. DOI: http://doi.org/10.6008/CBPC2179-684X.2021.001.0023 


\section{INTRODUÇÃO}

A Gestão da Cadeia de Suprimentos (GCS) corresponde à gestão de materiais e fluxos de informação dentro e entre as instalações, tais como fornecedores, plantas de fabricação e montagem e centros de distribuição (THOMAS et al., 1996). A cadeia de suprimento (CS) constitui uma rede de relações comerciais, em que o sucesso de um único negócio depende primariamente de sua capacidade de integrar sua rede de relacionamentos comerciais (SIMON et al., 2015).

Para promover uma gestão eficaz da CS existem diversos tipos de práticas a serem adotadas pelas empresas para atingirem suas metas. Muitas destas práticas possuem objetivos em comum e se distinguem por algumas particularidades como as práticas de integração, de coordenação, de alinhamento de objetivos, de relacionamento com clientes, de parcerias estratégicas com fornecedores, de filosofia Lean, de filosofia just-in-time, de estratégia de suprimentos, de gestão de qualidade total, de planejamento da demanda, de gestão de estoque e distribuição, de sistemas de informação, de melhoria contínua, de gestão logística e práticas sustentáveis/ambientais, dentre outras.

De acordo com Li et al. (2006), as práticas de GCS são um conjunto de atividades realizadas por uma organização para promover uma gestão eficaz da cadeia de fornecimento. Os autores apresentam as práticas de GCS como um conceito multidimensional, incluindo os lados a montante e a jusante da cadeia de fornecimento. Eles delimitam as práticas de GCS como uma construção de cinco dimensões: parceria estratégica com fornecedor, relacionamento com o cliente, nível de compartilhamento de informação, qualidade de compartilhamento de informações e postergação conforme demanda, que pode promover uma gestão eficaz.

Alguns estudos apontam que as práticas de GCS são implementadas pelas empresas com objetivo de obter uma vantagem competitiva (DEAN JUNIOR et al., 1994; GODFREY, 1993; SPITZER, 1993; NOVACK et al., 1995). Neste estudo, o termo utilizado será "diferencial competitivo", que possui a mesma definição de Porter (2011) para o termo anterior: “... sucesso do mercado em relação aos concorrentes devido à implementação de uma estratégia comercial eficaz".

Muitos autores também afirmam que as práticas de GCS melhoram o desempenho organizacional, tanto na performance de mercado quanto na performance financeira (ALVARADO et al., 2001; BALTACIOGLU et al., 2007; BOON-ITT et al., 2011; CHONG et al., 2011; CHO et al., 2012; DONLON, 1996; ELLRAM et al., 2007; LENNY-KOH et al., 2007; PETROVIC-LAZAREVIC et al., 2007; SUNDRAM et al., 2011; TAN et al., 1998).

Desta forma, este trabalho analisou as seguintes hipóteses: Hipótese 1: As práticas de GCS impactam positivamente no desempenho organizacional; Hipótese 2: As empresas com altos níveis de práticas de GCS terão altos níveis de diferencial competitivo; Hipótese 3: As empresas com altos níveis de diferencial competitivo terão altos níveis de desempenho organizacional.

Observando a escassez de literatura sobre o uso de práticas de GCS e seu efeito sobre o desempenho em economias emergentes de pequenas e médias empresas, verifica-se que ainda hoje no Brasil há uma carência de estudos em todos os portes de empresas e tipos de cadeias de suprimento quando se trata do 
tema. Assim, as principais contribuições deste trabalho é o estudo da relação entre as práticas de GCS com o diferencial competitivo e o desempenho organizacional em um dos setores mais importantes da economia de Minas Gerais.

A Seção 2 deste trabalho apresenta o referencial teórico enquanto a Seção 3 descreve a metodologia usada nesta pesquisa. Os resultados e análises são apresentados nas Seções 4 e 5. Por fim, a Seção 6 apresenta as implicações da pesquisa e suas limitações e a Seção 7 apresenta as conclusões desta pesquisa e propostas de trabalhos futuros.

\section{REVISÃO TEÓRICA}

\section{Gestão da cadeia de suprimentos}

Uma CS consiste em todas as partes envolvidas, direta ou indiretamente, na realização do pedido de um cliente. Os membros de uma cadeia de fornecimento incluem todas as empresas/organizações com as quais a empresa focal interage direta ou indiretamente por meio de seus fornecedores ou clientes, do ponto de origem ao ponto de consumo (LAMBERT et al., 2000). A CS inclui todas as funções envolvidas na recepção e na realização de uma solicitação do cliente, desenvolvimento de produto, marketing, operações, distribuição, finanças e serviços ao cliente (CHOPRA et al., 2011).

Os membros de uma cadeia de fornecimento incluem todas as empresas/organizações com as quais a empresa focal interage direta ou indiretamente por meio de seus fornecedores ou clientes, do ponto de origem ao ponto de consumo (LAMBERT et al., 2000).

Council of Logistics Management (CLM, 2000) define a GCS como a coordenação sistêmica e estratégica das funções de negócios tradicionais e como a coordenação tática através dessas funções em uma organização particular e em todos os negócios dentro da CS, melhorando o desempenho a longo prazo das organizações individuais e da CS como um todo.

Muitos estudiosos do campo de GCS salientam a importância da integração da mesma. Com o aumento da concorrência global, as organizações têm repensado a necessidade de cooperação e de melhoria dos processos, a fim de estabelecer relacionamentos mais próximos e integrados entre os elos da cadeia (FLYNN et al., 2010). Nesse sentido, a colaboração entre os participantes da cadeia mostra-se apropriada para otimizar os ganhos do arranjo (RODRIGUES et al., 2008).

\section{Práticas de gestão da cadeia de suprimentos}

Para Li et al. (2006) as práticas de GCS têm sido definidas como um conjunto de atividades realizadas em uma organização para promover a gestão eficaz de sua CS.

Os autores afirmam que existem várias práticas de gestão, mas tratam apenas de cinco dimensões que possibilitaram a mensuração no modelo proposto, que são: parceria estratégica com fornecedor, relacionamento com o cliente, nível de compartilhamento de informação, qualidade de compartilhamento de informações e postergação conforme demanda. 
Estudos anteriores indicaram que a parceria com fornecedor estratégico tem um impacto sobre vários aspectos do diferencial competitivo, como o preço/custo, em que essa parceria pode alavancar as capacidades estratégicas e operacionais das organizações participantes individualmente, ajudando-as a obter benefícios contínuos (LI et al., 2006; WOOK-KIM, 2006; LENNY-KOH et al., 2007; PETROVIC-LAZAREVIC et al., 2007; CHIOU et al., 2011; GHARAKHANI et al., 2012; ARDIANTO et al., 2013).

O termo é definido por Li et al. (2006) como a relação de longo prazo entre a organização e seus fornecedores, em que essas parcerias permitem que as organizações trabalhem mais eficazmente com alguns fornecedores importantes que estão dispostos a compartilhar a responsabilidade para o sucesso dos produtos.

Já o relacionamento com o cliente envolve o tratamento de reclamações, satisfação do cliente e criação de relacionamento de longo prazo (LENNY-KOH et al., 2007). Lambert et al. (2000) afirmam que todos na empresa devem ter foco no cliente, em que o conceito de marketing não se aplica apenas para o departamento de marketing, mas responsabiliza toda a empresa em se concentrar no atendimento das necessidades do cliente. Segundo eles, todos os executivos gostariam de gerenciar suas cadeias de suprimentos até o ponto de consumo, porque quem tem a relação com o usuário final tem o poder na CS.

O nível de compartilhamento de informações é uma prática de gestão que envolve até que ponto as informações críticas da empresa são comunicadas ao parceiro da CS, enquanto a qualidade da troca de informações refere-se à precisão, oportunidade, adequação e credibilidade das informações trocadas (LI et al., 2006). Segundo Choi et al. (2001), as empresas devem buscar maximizar o seu lucro individual através da troca de informações, considerando os fluxos de conhecimento e não apenas dados.

O compartilhamento de informações com qualidade significa que as informações comunicadas entre parceiros precisam ter precisão, adequação e gerar oportunidade (LENNY-KOH et al., 2007).

Alderson (2006) criou o termo postergação, para definir o ato de adiar o máximo possível a movimentação e/ou conclusão de produtos e serviços no processo produtivo ou de distribuição. Desta forma o produto só é deslocado se a localização da demanda já estiver determinada, ao mesmo tempo que a conclusão de produtos e serviços só acontece quando as preferências do consumidor são conhecidas. Ele define que a postergação envolve desde projetar e desenvolver produtos que possam ser customizados rapidamente e a baixo custo a partir da demanda do consumidor até a implementação de estratégias de estoque em canal de distribuição para o momento exato do pedido.

\section{Diferencial competitivo}

Li et al. (2006) destacam que o diferencial competitivo está relacionado com as habilidades competitivas das empresas para se diferenciarem dos seus concorrentes. Baseando-se em diversas literaturas, os autores determinaram como dimensões para a construção de diferencial competitivo os itens utilizados neste estudo: preço/custo, qualidade, tempo para introduzir novos produtos no mercado, inovação de produtos e confiabilidade de entrega.

As práticas de GCS podem impactar positivamente na redução dos custos de produção, uma vez que 
se alcança uma otimização de recursos, como na redução de tempo de ciclo de produção, redução de estoque, evitando desperdícios. Li et al. (2006) propõem medir a variável preço/custo através das variáveis que indicam se as empresas oferecem preços competitivos e se são capazes de oferecer preços tão baixos ou menores do que os concorrentes.

Lenny-Koh et al. (2007) afirmam que a qualidade dos produtos e serviços vendidos estão diretamente relacionados com o custo e a qualidade dos bens e serviços adquiridos. A qualidade será medida neste trabalho observando se uma organização é capaz de oferecer qualidade no produto/serviço de forma que está crie valor superior ao dos concorrentes. Desta forma, Li et al. (2006) propõem o uso de variáveis referentes a confiança da empresa sobre a competitividade através da qualidade e variáveis referentes à confiabilidade, durabilidade e alta qualidade de produtos/serviço para o cliente.

Cohen et al. (1996) explicam que a redução do tempo de ciclo de desenvolvimento de novos produtos e melhorias no desempenho do produto tornaram-se objetivos estratégicos para muitas empresas. Eles acrescentam que uma melhoria na velocidade de desenvolvimento do produto não conduz necessariamente a um tempo de lançamento mais precoce, mas na maioria das vezes leva a produtos melhorados. Nesta variável, este trabalho procurou analisar o tempo para o mercado (time-to-market), ou seja, se uma organização é capaz de introduzir novos produtos mais rapidamente do que os principais concorrentes.

Lambert et al. (2000) destacam que novos produtos são a alma de uma empresa, mas que os produtos certos devem ser desenvolvidos e lançados com sucesso em prazos cada vez mais curtos, a fim de se manterem competitivas. Assim, os gestores devem trabalhar no sentido de desenvolver tecnologia de produção em fluxo de produção para fabricar e integrar o melhor da CS para fluir a combinação produto/mercado.

A confiabilidade da entrega ocorre quando se consegue entregar os pedidos no prazo certo, que corresponde a um prazo aceito pelo cliente e aceito pelo mercado comparando-se com a entrega dos concorrentes (SLACK, 2002). Andrade et al. (2011) destacam que os objetivos da confiabilidade de entrega são: desenvolver estratégias e alternativas que permitam à empresa entregar o produto sempre no prazo definido, respeitando os prazos de entrega estabelecidos pelos clientes. Para atingir os objetivos é necessário investir no treinamento de funcionários e motoristas que realizam a operação de entrega do produto, podese criar um setor de planejamento de entrega e produção, a fim de aumentar a assertividade e até mesmo investir em um software de programação de entrega.

\section{Desempenho organizacional: performance de mercado e financeira}

Robb et al. (2008) defendem que as práticas de operações na CS influenciam positivamente no desempenho de mercado e Lenny-Koh et al. (2007) acrescentam que o aumento do desempenho operacional pode levar a altos níveis de desempenho organizacional relacionado com GCS em termos de aumento das vendas e a coordenação de toda a organização e integração da CS.

Van Der Vaart et al. (2008) frisam que o desempenho deve ser medido separadamente conforme o poder que a empresa tem dentro da cadeia. Eles destacam que é necessário saber o posicionamento da 
empresa na cadeia, tanto a jusante quanto a montante ou até mesmo se exercem alguma governança.

Li et al. (2006) definem o desempenho organizacional no sentido de quão bem uma organização atinge metas orientadas para o mercado, bem como metas financeiras, analisando a quota de mercado, o retorno do investimento, o crescimento da quota de mercado, o crescimento das vendas, o crescimento sobre o investimento, a margem de lucro sobre as vendas e a posição competitiva geral.

\section{Pesquisas similares}

Pesquisas anteriores testaram hipóteses similares deste estudo verificando a correlação entre multivariáveis, considerando o impacto das práticas da gestão da cadeia de suprimentos sobre o diferencial competitivo ou considerando o impacto das práticas sobre o desempenho organizacional. Os únicos estudos que fizeram a análise sobre ambas variáveis - diferencial competitivo e desempenho organizacional - foram Wook-Kim (2006), Green Junior et al. (2006) e Li et al. (2006).

Nos Estados Unidos da América, Gowen et al. (2003) estudaram o impacto das práticas da GCS apenas sobre o diferencial competitivo entrevistando 358 empresas no setor de manufatura e serviços. Nesta mesma linha, os pesquisadores Chiou et al. (2011) entrevistaram 124 indústrias de médio e grande porte em Taiwan e Bratić (2011) entrevistou 150 empresas gráficas na Croácia. Ambas as pesquisas atestaram que as práticas da GCS têm impacto positivo direto no diferencial competitivo.

Já na Turquia, uma pesquisa similar - aplicada em 203 micro e pequenas empresas de manufatura concluiu que as práticas de GCS têm impacto direto positivo e significativo no desempenho organizacional (LENNY-KOH et al., 2007). Outros autores também fizeram pesquisa observando impacto apenas sobre o desempenho organizacional em outros países chegando à mesma conclusão, como Petrovic-Lazarevic et al. (2007), Lenny-Koh et al. (2007), Khang et al. (2010), e Chong et al. (2011) - Quadro 1.

No ano de 2006, três pesquisas foram publicadas observando o impacto das práticas da GCS tanto sobre o diferencial competitivo quanto sobre o desempenho organizacional. Nos Estados Unidos da América, Li et al. (2006) entrevistaram 196 empresas de grande porte nos setores, móveis e utensílios, borracha e plásticos, produtos metálicos, indústria e comércio de máquinas, equipamentos eletrônicos e transporte de equipamento e os resultados desta investigação apoiaram a visão de que as práticas de GCS podem impactar o diferencial competitivo e desempenho organizacional das empresas. Green Junior et al. (2006) entrevistaram 80 empresas de grande porte confirmando as mesmas hipóteses usando variáveis diferentes.

Já no Japão e na Coréia, Wook-Kim (2006), obteve apenas um resultado diferente de Green Junior et al. (2006) e Li et al. (2006) para as empresas de pequeno porte, em que os resultados indicaram que as práticas de GCS e o diferencial competitivo poderiam não estar relacionados diretamente com o desempenho organizacional e, portanto, precisariam de mecanismos intermediários para ligações eficientes entre as variáveis. Já em empresas de grande porte o resultado das hipóteses de impacto das práticas de GCS foi positivo e direto sobre o diferencial competitivo e sobre o desempenho organizacional. 
Quadro 1: Pesquisas Similares.

\begin{tabular}{|c|c|c|c|c|c|c|c|}
\hline Países & Ano & Autores & Observações & $\begin{array}{l}\text { Seguimento das } \\
\text { empresas }\end{array}$ & $\begin{array}{l}\text { Práticas GCS } \\
\text { X D.O }\end{array}$ & $\begin{array}{l}\text { Práticas GCS } \\
\text { X D.C }\end{array}$ & $\begin{array}{l}\text { Hipótese } \\
\text { Confirm. }\end{array}$ \\
\hline USA & 2003 & Gowen et al. & 358 empresas & manufatura e serviço & & $x$ & $\operatorname{sim}$ \\
\hline $\begin{array}{l}\text { Japão } \quad \text { e } \\
\text { Coréia }\end{array}$ & 2006 & Wook-Kim & 623 empresas & indústria Manufatura & $x$ & $x$ & sim/não \\
\hline USA & 2006 & $\begin{array}{l}\text { Green Junior et } \\
\text { al. }\end{array}$ & 80 empresas & grande porte & $x$ & $x$ & $\operatorname{sim}$ \\
\hline USA & 2006 & Li et al. & 196 empresas & $\begin{array}{l}\text { grande porte de } \\
\text { diversos setores }\end{array}$ & $x$ & $x$ & $\operatorname{sim}$ \\
\hline Austrália & 2007 & $\begin{array}{l}\text { Petrovic- } \\
\text { lazarevic et al. }\end{array}$ & 64 empresas & indústria Manufatura & $x$ & & $\operatorname{sim}$ \\
\hline Turquia & 2007 & Lenny-Koh et al. & 203 empresas & micro e pequenas & $x$ & & $\operatorname{sim}$ \\
\hline Malásia & 2010 & Khang et al. & 246 empresas & serviços Industriais & $x$ & & $\operatorname{sim}$ \\
\hline Malásia & 2011 & Chong et al. & 163 empresas & manufatura e serviços & $\mathrm{x}$ & & $\operatorname{sim}$ \\
\hline Croácia & 2011 & Bratić. & 150 empresas & empresas gráficas & & $x$ & $\operatorname{sim}$ \\
\hline Taiwan & 2011 & Chiou et al. & 124 empresas & $\begin{array}{l}\text { indústrias médio e } \\
\text { grande porte }\end{array}$ & & $x$ & $\operatorname{sim}$ \\
\hline Iran & 2012 & Gharakhani et al. & 186 empresas & manufatura e serviço & $x$ & & $\operatorname{sim}$ \\
\hline Indonésia & 2013 & Ardianto et al. & 132 empresas & $\begin{array}{l}\text { grande porte (apenas } \\
\text { de } 1 \text { estado) }\end{array}$ & $x$ & & $\operatorname{sim}$ \\
\hline
\end{tabular}

\section{METODOLOGIA}

Este trabalho se classifica como uma pesquisa aplicada quanto à natureza, descritiva quanto ao objetivo e quantitativa em que os dados serão mensurados através de Modelagem de Equações Estruturais.

Modelagem de Equações Estruturais vem do termo inglês Structural Equation Modeling (SEM) e para Hair Junior et al. (2009) está modelagem procura explicar as relações entre múltiplas variáveis, examinando a estrutura de inter-relações demonstradas em uma série de equações que descrevem todas as relações entre construtos - variáveis dependentes e independentes - envolvidos na análise. Uma das características básicas da SEM é que se pode testar uma teoria de ordem causal entre um conjunto de variáveis (FARIAS et al., 2000). As práticas de GCS de Li et al. (2006) foram concebidas como um modelo de segunda ordem composto por cinco dimensões descritas na Seção 2.2 deste trabalho, utilizando SEM para determinar se um modelo de fatores de ordem superior é apropriado para as práticas de GCS.

A modelagem quantifica de que forma as variáveis observadas podem ser indicativos indiretos de variáveis latentes, que são conhecidas como construtos ou fatores. Neste trabalho usa-se os fatores 'parceria estratégica com fornecedor', 'postergação conforme demanda', 'compartilhamento de informação', 'qualidade da informação compartilhada', 'relação com os clientes' para a variável 'práticas de GCS' e para a variável 'diferencial competitivo' foram usados os construtos: 'preço|custo', 'qualidade dos produtos', 'produtos inovadores', 'confiabilidade de entrega' e 'tempo de desenvolvimento de novos produtos para o mercado'.

Já a variável 'desempenho organizacional' é medida através dos construtos relacionados ao market share e objetivos financeiros. O desempenho organizacional também pode ser representado como um construto de segunda ordem, uma vez que se pode utilizar os construtos desempenho organizacional e desempenho financeiro como realizado no trabalho de Li et al. (2006). O mesmo pode ocorrer, por exemplo, com o diferencial competitivo utilizando construtos da variável preço/custo e qualidade.

As relações entre variáveis podem ser descritas em correlação, que indica o grau de linearidade entre 
duas variáveis de covariância, dando a medida de quanto duas variáveis variam juntas (COLLARES et al., 2012). Assim temos o modelo de mensuração, que é a designação de variáveis indicadoras de construtos representados em um diagrama e o modelo estrutural, que demonstra as relações de dependência entre os construtos (HAIR JUNIOR et al., 2009).

Assim, o survey foi aplicado em empresas do setor metalmecânico de Minas Gerais, que é uma das principais atividades econômicas do estado (BANDES, 2005).

\section{População, amostra e coleta de dados}

A população foi de 1850 empresas do setor metalmecânico, e a amostra final totalizou 269 respondentes com questionários válidos, o que para Hair Junior et al. (2009) é uma quantidade suficiente para a análise dos dados, considerando o survey e a modelagem de equações estruturais em que é sugerido amostras em torno de 200 a 400 elementos ou de 10 a 15 respondentes por variável. Segundo Hair Junior et al. (2009), tamanho excessivo de amostra poderia comprometer a análise de significância em testes de hipóteses.

\section{Avaliação do modelo de mensuração e estrutural}

A avaliação do modelo de mensuração - das variáveis indicadas para os construtos - ocorreu através de diversos índices de qualidade de ajuste (goodness index - GOF), que indicam a validade do modelo. As medidas de ajuste utilizadas foram: RMSEA, GFI, AGFI, e CFI, conforme Quadro 2.

Quadro 2: Índice de qualidade de ajuste.

\begin{tabular}{|l|l|}
\hline ÍNDICES & Valores aceitáveis \\
\hline GOF chi-quadrado $(\mathrm{P})$ & Quanto menor e não significativo, melhor. \\
\hline RMSEA & $<0,10$ \\
\hline GFI & $>0,90$ \\
\hline AGFI & $<\mathrm{GFI}$ \\
\hline CFI & $>0,90$ \\
\hline
\end{tabular}

Fonte: Hair Junior et al. (2009).

\section{RESULTADOS E DISCUSSÃO}

\section{Dados demográficos}

Das respostas obtidas, $46,8 \%$ são de indústrias e $13 \%$ de comércio/serviço consideradas microempresas pelo número de funcionários, segundo classificação SEBRAE, $46 \%$ tem a receita de 1 a 10 milhões por ano e $41 \%$ tem vendas abaixo de 1 milhão.

Apesar da diversidade de atividades de empresas do setor metalmecânico, as entidades respondentes concentraram-se em 24521 - Fundição de metais não-ferrosos e suas ligas (17\%); 25110 Fabricação de estruturas metálicas (23\%); 25136 - Fabricação de obras de caldeiraria pesada (14\%); e 25390 - Serviços de usinagem, solda, tratamento e revestimento em metais (19\%).

Dos respondentes, $58 \%$ possuem cargos de tomada de decisão - CEO/Presidente/Vice-presidente (11\%), Diretor (16\%) e Gerente (31\%) - e os outros $42 \%$ foram de demais cargos. $31 \%$ dos tomadores de 
decisão possuíam mais de 10 anos de trabalho nas empresas e 15 \% de 6 a 10 anos. Esses dados demonstram que, de modo geral, os respondentes eram qualificados em termos de função para fornecer informações úteis para a pesquisa.

\section{Modelagem de equações estruturais}

Inicialmente, apresenta-se os índices de ajuste sugeridos por Hair Junior et al. (2009) e utilizados por Li et al. (2006) na Tabela 1. Neste trabalho utilizou-se o software IBM ${ }^{\circledR}$ SPSS AMOS ${ }^{\circledR}$ no modelo SEM estudado.

Tabela 1: Índice de qualidade de ajuste.

\begin{tabular}{lllll}
\hline \hline Índices & GCS & Dif. Comp. & Desemp. Op. & Valores Bons \\
\hline GOF (P) & 0,696 & 0,000 & 0,000 & 0,000 \\
RMSEA & 0,000 & 0,06 & 0,106 & $<0,10$ \\
GFI & 0,995 & 0,925 & 0,942 & $>0,90$ \\
AGFI & 0,986 & 0,893 & 0,885 & $<$ GFI \\
CFI & 1,0 & 0,957 & 0,973 & $>0,90$ \\
\hline \hline
\end{tabular}

Observando o índice RMSEA, verifica-se que apenas o construto Desempenho Organizacional ficou centésimos acima do limite de ajuste, o que é aceitável pela diferença insignificante. O índice GFI foi ótimo em todos os construtos e o $\mathrm{AGFI}$ teve resultados razoáveis em todos os construtos, por terem valores menores que o GFI - segundo Hair Junior et al. (2009), o AGFI deve ser menor que o GFI em proporção a complexidade do modelo.

Optou-se por não fazer quaisquer modificações adicionais ao modelo de Li et al. (2006), uma vez que o único índice ruim foi $x^{2}$ 0,696 do constructo da GCS que não pode ser analisado isoladamente (HAIR JUNIOR et al., 2009). A manutenção do modelo de mensuração foi priorizada objetivando o ajuste teórico ao invés do ajuste empírico (OLSSON et al., 2000).

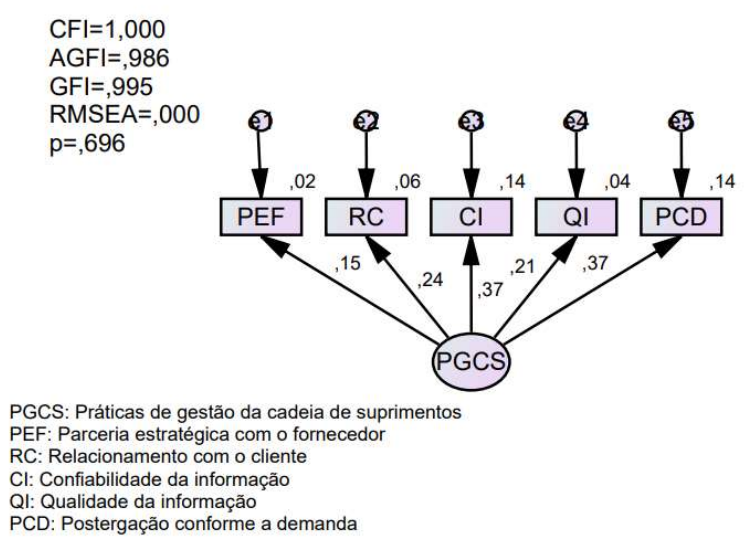

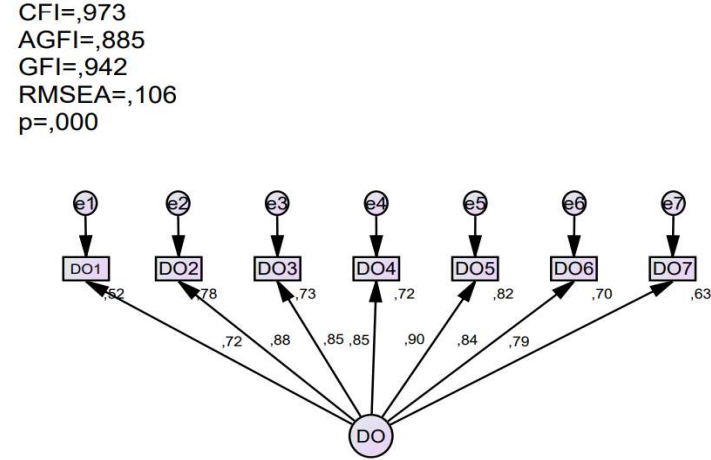

Figura 2: Desempenho Organizacional.

Figura 11: Práticas de GCS.

\section{Modelo de diferencial competitivo}

Devido aos erros que ocorreram durante a rotação dos dados no modelo SEM, foi necessário rodar o grau de confiabilidade - Alfa de Cronbach - dos resultados obtido.

Segundo Streiner (2003), o valor mínimo aceitável para o alfa Cronbach é 0,70 e o valor máximo 
esperado é 0,90, acima deste valor considera-se que há redundância ou duplicação, dessa forma, o autor sugere que os itens redundantes e variáveis de baixa confiabilidade devem ser eliminados. Li et al. (2006) cita que algumas questões também foram eliminadas do modelo SEM de sua pesquisa.

Conforme Tabela 2, os dados da variável Preço/Custo não apresentam grau de confiabilidade e foram eliminados no modelo SEM. Uma das causas da baixa confiabilidade da variável Preço/Custo pode ser o número de questões do formulário, uma vez que Hair Junior et al. (2009) sugere no mínimo 3 questões por variável e Li et al. (2006) construiu o questionário com 2 questões.

Tabela 2: Grau de confiabilidade - Alfa de Cronbach.

\begin{tabular}{llll}
\hline \hline Variáveis & Alfa de Cronbach & Fator de 2a ordem & No de itens \\
\hline Preço/custo &, 385 & DCPC1; DCPC2 & 2 \\
Qualidade Produto/Serviço &, 807 & DCQL1; DCQL2; DCQL3; DCQL4 & 4 \\
Confiabilidade da entrega &, 812 & DCCE1; DCCE2; DCCE3 & 3 \\
Inovação &, 761 & DCIP1; DCIP2; DCIP3 & 3 \\
Tempo para mercado &, 887 & DCTM1; DCTM2; DCTM3; DCTM4 & 4 \\
\hline \hline
\end{tabular}

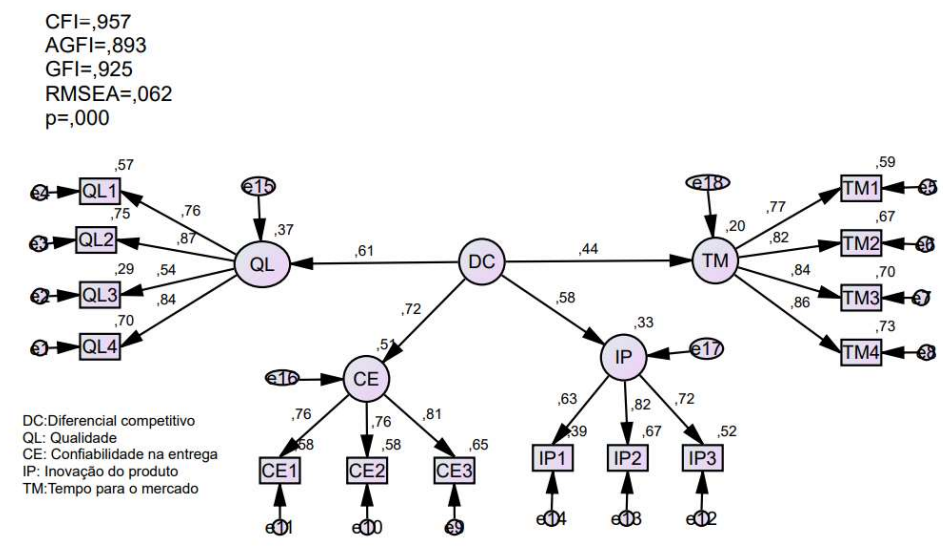

Figura 33: Diferencial Competitivo.

\section{Modelos estruturais}

A Figura 4 apresenta os resultados das hipóteses estudadas neste trabalho. Neste caso, o coeficiente normalizado da Hipótese 1 é de $-0,01$ para $\mathrm{P}=000$, mostrando que o impacto das práticas de GCS sobre o desempenho organizacional é neutro. A implementação das práticas de GCS neste trabalho demonstrou não impactar diretamente o desempenho financeiro e o de mercado de uma organização.

Resultado semelhante aconteceu em uma pesquisa aplicada no Japão e na Coréia, em que os dados demonstraram que não houve impacto das práticas de GCS sobre o desempenho organizacional para empresas de pequeno e médio porte, enquanto nas empresas de grande porte o resultado das hipóteses de impacto das práticas de GCS foi positivo e direto sobre o desempenho organizacional (WOOK-KIM, 2006).

Como o perfil da maioria das empresas entrevistadas foi de micro e pequenas empresas, pode-se levar em consideração que as práticas de GCS não impactam no desempenho organizacional de empresas com este perfil. A hipótese 2 é suportada, indicando que as práticas GCS têm um impacto direto no diferencial competitivo. O coeficiente normalizado é 0,04 que é estatisticamente significativo a $\mathrm{P}=0,00$.

A hipótese 3 também é confirmada conforme Figura 4, em que os resultados demonstram que níveis mais elevados de diferencial competitivo podem levar a um desempenho organizacional melhorado. $\mathrm{O}$ 
coeficiente normalizado também é 0,04 que é estatisticamente significativo a $P=0,00$. Com base nos coeficientes padronizados das três hipóteses apresentadas na Tabela 3, as práticas da GCS podem ter maior impacto na diferencial competitivo $(=0,041)$ do que no desempenho organizacional $(=-0,008)$.

O resultado também demonstra que o desempenho organizacional é mais influenciado pelo diferencial competitivo $(=0,04)$ do que pelas práticas de GCS $(=-0,01)$. O coeficiente padronizado do efeito indireto das práticas de GCS no desempenho organizacional é de 0,002. Assim, as práticas de GCS não têm uma influência direta no desempenho organizacional, mas influencia indiretamente através do diferencial competitivo - Tabela 3.

Tabela 3: Hipóteses testadas.

\begin{tabular}{|c|c|c|c|c|c|}
\hline Hipótese & Relação & Efeitos Totais & Efeitos Diretos & Efeitos Indiretos & Hipótese \\
\hline $\mathrm{H} 1$ & Prat. GCS $\rightarrow$ DO & $-0,007$ & $-0,008$ & 0,002 & Não Suportada \\
\hline $\mathrm{H} 2$ & Prat. GCS $\rightarrow$ DC & 0,041 & 0,041 & & Suportada \\
\hline õesH3 & $\mathrm{DC} \rightarrow \mathrm{DO}$ & 0,039 & 0,039 & & Suportada \\
\hline
\end{tabular}

Como no trabalho de Li et al. (2006), este trabalho indicou que o diferencial competitivo é uma medida intermediária entre as práticas de GCS e o desempenho organizacional, em que este desempenho só é melhorado pelas práticas de GCS se produzirem diferencial competitivo.

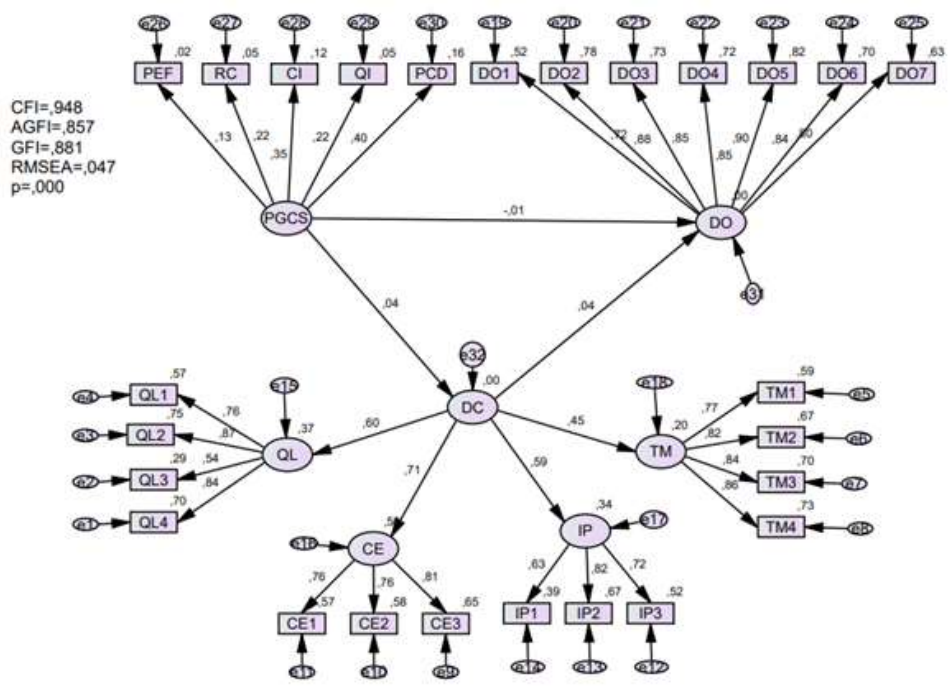

Figura 4: Hipóteses.

\section{Implicações da pesquisa e limitações}

O presente estudo, buscou aplicar o questionário de Li et al. (2006) sobre as práticas de GCS, demonstrando eficácia na melhoria do desempenho organizacional e o diferencial competitivo. Ao contrário do trabalho de Li et al. (2006), o presente estudo teve um construto de segunda ordem apenas para o diferencial competitivo. Através da análise da relação de construção das práticas de GCS com o diferencial competitivo (Hipótese 1), foi demonstrado que as práticas de GCS pode impactar diretamente no diferencial competitivo, o que aponta para a importância de práticas de GCS para a organização. Os resultados desta investigação apoiam que as práticas de GCS têm um impacto direto sobre o diferencial competitivo e indireto sobre o desempenho organizacional. 
Li et al. (2006) destacam que as práticas de GCS podem ser influenciadas por fatores contextuais, como o tipo de indústria, tamanho da empresa, posição da empresa na CS, tamanho da CS e o tipo de CS.

Este estudo teve um número limitado de observações (269) e um grande número de micro e pequenas empresas, mas observou uma região específica como sugerido por Li et al. (2006). Estudos futuros ainda podem testar os relacionamentos/dependências entre cinco dimensões das práticas de GCS e dos outros construtos. Os dados do estudo foram coletados apenas com um único respondente das organizações, o que pode ser uma causa para um possível viés de resposta, podendo gerar alguma imprecisão de medição.

Pesquisas futuras podem coletar dados de vários entrevistados de cada organização participante (médio e grande porte) para aprimorar os resultados da pesquisa. Pesquisas futuras também podem comparar o resultado deste estudo analisando respostas do Arranjo Produtivo Local Metalmecânico localizado no mesmo estado para observar as práticas de GCS e os outros construtos.

\section{CONCLUSÕES}

Esta pesquisa empírica levantou o perfil das empresas pesquisadas do setor metalmecânico de Minas Gerais, que foram caracterizadas, em sua maioria, como indústrias de micro e pequeno porte, com atividades preponderantes de fabricação de estruturas não metálicas, fundição de metais não ferrosos e fabricação de obras de caldeiraria pesada.

A prática de GCS mais usada por essas empresas em relação aos fornecedores foi a busca pela qualidade, e quanto ao cliente foi a busca por interação para estabelecer confiabilidade. Com relação ao nível de compartilhamento de informações a maioria respondeu que informa com antecedência aos parceiros comerciais sobre mudanças de necessidade e que a troca de informação é feita no momento adequado. A maioria das empresas postergam as atividades finais de montagem do produto até que o pedido do cliente tenha sido efetivado

O diferencial competitivo foi analisado quanto à qualidade, confiabilidade de entrega, inovação de produtos e tempo para o mercado. Já o desempenho organizacional foi estudado através da quota de mercado, retorno sobre o investimento, o crescimento da quota de mercado, crescimento das vendas, crescimento sobre o retorno sobre o investimento, a margem de lucro sobre as vendas e posição competitiva geral.

Foram analisadas três hipóteses de pesquisa: (1) Empresas com altos níveis de práticas de GCS têm altos níveis de desempenho organizacional; (2) Empresas com elevado nível de práticas de GCS têm altos níveis de diferencial competitivo; (3) Empresas com altos níveis de diferencial competitivo têm um alto nível de desempenho organizacional.

Para testar as hipóteses foi aplicado um questionário de Li et al. (2006) considerado válido e confiável para avaliar as práticas de GCS. O survey foi testado estatisticamente e os construtos de primeira e segunda ordem foram validados.

Os resultados indicam que os níveis mais altos de práticas de GCS podem levar ao diferencial competitivo melhor, mas não teve impacto sobre o desempenho organizacional. Além disso, o diferencial 
competitivo pode ter um impacto direto e positivo sobre o desempenho organizacional. Este estudo fornece evidência empírica para sustentar conceitos sobre o impacto das práticas de GCS encontrados na literatura.

\section{REFERÊNCIAS}

ALDERSON, W.. Marketing efficiency and the principle of postponement. In: A twenty-first century guide to Aldersonian marketing thought. Boston: Springer, 2006. p.109-113.

ALVARADO, U. Y.; KOTZAB, H.. Supply chain management: the integration of logistics in marketing. Industrial Marketing Management, v.30, n.2, p.183-198, 2001.

ARDIANTO, Y. T.; SURACHMAN, U. S.; ZAIN, D.. An Empirical Internal Perceptions Study of the Implementation Supply Chain Management in Indonesian Manufacturing Companies As a Mediating Factor of Information Technology Utilization to Organization Performances. European Journal of Business and Management, v.5, n.16, 2013.

ANDRADE, M.; JUNQUEIRA, A. G. W.. Gestão da Produção: Utilização da matriz importância-desempenho em uma indústria de rações para aves. Revista Destaques Acadêmicos, v.2, n.1, 2011.

BALTACIOGLU, T.; ADA, E.; KAPLAN, M. D.; AND, O. Y.; KAPLAN, Y. C.. A new framework for service supply chains. The Service Industries Journal, v.27, n.2, p.105-124, 2007.

BANDES. Plano de Desenvolvimento do Arranjo Metalmecânico Capixaba. BANDES, 2005.

BRATIĆ, D.. Achieving a competitive advantage by SCM. IBIMA Business Review, v.2011, p.1-13, 2011.

BOON-ITT, S.; PONGPANARAT, C.. Measuring service supply chain management processes: The application of the Q-sort technique. International Journal of Innovation, Management and Technology, v.2, n.3, p.217, 2011.

CHIOU, T. Y.; CHAN, H. K.; LETTICE, F.; CHUNG, S. H.. The influence of greening the suppliers and green innovation on environmental performance and competitive advantage in Taiwan. Transportation Research Part E: Logistics and Transportation Review, v.47, n.6, p.822-836, 2011.

CHO, D. W.; LEE, Y. H.; AHN, S. H.; HWANG, M. K.. A framework for measuring the performance of service supply chain management. Computers \& Industrial Engineering, v.62, n.3, p.801-818, 2012

CHOI, T. Y.; DOOLEY, K. J.; RUNGTUSANATHAM, M.. Supply networks and complex adaptive systems: control versus emergence. Journal of Operations Management, v.19, n.3, p.351-366, 2001.

CHONG, A. Y.; CHAN, F. T.; OOI, K. B.; SIM, J. J.. Can Malaysian firms improve organizational/innovation performance via SCM?. Industrial Management \& Data Systems, v.111, n.3, p.410-431, 2011.

CHOPRA, S.; MEINDL, P.. Gestão da cadeia de suprimentos: estratégia, planejamento e operações. Pearson, 2011.
COHEN, M. A.; ELIASBERG, J.; HO, T. H.. New product development: The performance and time-to-market tradeoff. Management Science, v.42, n.2, p.173-186, 1996.

COLLARES, C. F.; GREC, W. L. P.; MACHADO, J. L. M.. Psicometria na garantia de qualidade da educação médica: conceitos e aplicações. Sci. Health, v.3, n.1, p.33-49, 2012.

CLM. Council of Logistics Management. What it's all about. Oak Brook: CLM, 2000.

DEAN JUNIOR, J. W.; BOWEN, D. E.. Management theory and total quality: improving research and practice through theory development. Academy of Management Review, v.19, n.3, p.392-418, 1994.

DONLON, J. P.. Maximizing value in the supply chain. Chief Executive, v.117, n.1, p.54-63, 1996.

ELLRAM, L. M.; TATE, W. L.; BILLINGTON, C.. Services supply management: The next frontier for improved organizational performance. California Management Review, v.49, n.4, p.44-66, 2007.

FARIAS, S. A. D.; SANTOS, R. D. C.. Modelagem de equações estruturais e satisfação do consumidor: uma investigação teórica e prática. Revista de Administração Contemporânea, v.4, n.3, p.107-132, 2000.

FLYNN, B. B.; HUO, B.; ZHAO, X.. The impact of supply chain integration on performance: A contingency and configuration approach. Journal of Operations Management, v.28, n.1, p.58-71, 2010.

GHARAKHANI, D.; MAVI, R. K.; HAMIDI, N.. Impact of supply chain management practices on innovation and organizational performance in Iranian Companies. African Journal of Business Management, v.6, n.19, p.5939-5949, 2012.

GODFREY, A. B.. Ten areas for future research in total quality management. Quality Management Journal, v.1, n.1, p.4770, 1993

GOWEN III, C. R.; TALLON, W. J.. Enhancing supply chain practices through human resource management. Journal of Management Development, v.22, n.1, p.32-44, 2003.

GREEN JUNIOR, K. W.; MCGAUGHEY, R.; CASEY, K. M.. Does supply chain management strategy mediate the association between market orientation and organizational performance?. Supply Chain Management: An International Journal, v.11, n.5, p.407-414, 2006.

HAIR JUNIOR, J. F.; BLACK, W. C.; BABIN, B. J.; ANDERSON, R. E.; TATHAM, R. L.; SANT'ANNA, M. A. G. A. S.. Análise multivariada de dados. Bookman, 2009. 
KHANG, T. S.; ARUMUGAM, V.; CHONG, A. Y. L.; CHAN, F. T. Relationship between supply chain management practices and organisation performance: a case study in the Malaysian service industry. International Journal of Modelling in Operations Management, v.1, n.1, p.84-106, 2010.

LAMBERT, D. M.; COOPER, M. C.. Issues in supply chain management. Industrial Marketing Management, v.29, n.1, p.65-83, 2000.

LENNY-KOH, S. C.; DEMIRBAG, M.; BAYRAKTAR, E.; TATOGLU, E.; ZAIM, S.. The impact of supply chain management practices on performance of SMEs. Industrial Management \& Data Systems, v.107, n.1, p.103-124, 2007.

LI, S.; RAGU-NATHAN, B.; RAGU-NATHAN, T. S.; RAO, S. S.. The impact of supply chain management practices on competitive advantage and organizational performance. Omega, v.34, n.2, p.107-124, 2006.

NOVACK, R. A.; LANGLEY JUNIOR, C. J.; RINEHART, L. M.. Creating logistics value: themes for the future. 1995.

OLSSON, U. H.; FOSS, T.; TROYE, S. V.; HOWELL, R. D.. The performance of $\mathrm{ML}, \mathrm{GLS}$, and WLS estimation in structural equation modeling under conditions of misspecification and nonnormality. Structural Equation Modeling, v.7, n.4, p.557595, 2000.

PETROVIC-LAZAREVIC, S.; SOHAL, A.; BAIHAQI, I.. Supply chain management practices and supply chain performance in the Australian manufacturing industry. Monash University Faculty of Business and Economics, 2007.

PORTER, M. E.. Competitive advantage of nations: creating and sustaining superior performance. Simon and Schuster, 2011.

ROBB, D. J.; XIE, B.; ARTHANARI, T.. Supply chain and operations practice and performance in Chinese furniture manufacturing. International Journal of Production Economics, v.112, n.2, p.683-699, 2008.

RODRIGUES, D. M.; SELLITTO, M. A.. Práticas logísticas colaborativas: o caso de uma cadeia de suprimentos da indústria automobilística. Revista de AdministraçãoRAUSP, v.43, n.1, p.97-111, 2008.

SIMON, A. T.; SERIO, L. C. D.; PIRES, S. R. I.; MARTINS, G. S.. Evaluating supply chain management: A methodology based on a theoretical model. Revista de Administração Contemporânea, v.19, n.1, p.26-44, 2015.

SPITZER, R. D.. TQM: the only source of sustainable competitive advantage. Quality Progress, v.26, p.59-59, 1993.

SLACK, N.. Vantagem competitiva em manufatura: atingindo competitividade nas operações industriais. Atlas, 2002.

STREINER, D. L.. Being inconsistent about consistency: When coefficient alpha does and doesn't matter. Journal of Personality Assessment, v.80, n.3, p.217-222, 2003.

SUNDRAM, V. P. K.; IBRAHIM, A. R.; GOVINDARAJU, V. G. R. Supply chain management practices in the electronics industry in Malaysia: Consequences for supply chain performance. Benchmarking: An International Journal, v.18, n.6, p.834-855, 2011.

TAN, K. C.; KANNAN, V. R.; HANDFIELD, R. B.. Supply chain management: supplier performance and firm performance. Journal of Supply Chain Management, v.34, n.3, p.2, 1998.

THOMAS, D. J.; GRIFFIN, P. M.. Coordinated supply chain management. European Journal of Operational Research, v.94, n.1, p.1-15, 1996.

VAN DER VAART, T.; VAN DONK, D. P.. A critical review of survey-based research in supply chain integration. International Journal of Production Economics, v.111, n.1, p.42-55, 2008.

WOOK-KIM, S.. Effects of supply chain management practices, integration and competition capability on performance. Supply Chain Management: An International Journal, v.11, n.3, p.241-248, 2006.

A CBPC - Companhia Brasileira de Produção Científica (CNPJ: 11.221.422/0001-03) detém os direitos materiais desta publicação. Os direitos referem-se à publicação do trabalho em qualquer parte do mundo, incluindo os direitos às renovações, expansões e disseminações da contribuição, bem como outros direitos subsidiários. Todos os trabalhos publicados eletronicamente poderão posteriormente ser publicados em coletâneas impressas sob coordenação da Sustenere Publishing, da Companhia Brasileira de Produção Científica e seus parceiros autorizados. Os (as) autores (as) preservam os direitos autorais, mas não têm permissão para a publicação da contribuição em outro meio, impresso ou digital, em português ou em tradução. 